\title{
Prevalência de diabetes mellitus em pacientes renais crônicos sob hemodiálise em Porto Alegre, Brasil
}

\author{
Prevalence of diabetes mellitus in chronic renal failure patients
} under haemodialysis in Porto Alegre, Brazil

\section{Autores}

Jayme Eduardo

Burmeister $^{1}$

Camila Borges

Mosmann ${ }^{2}$

Renata $\mathrm{Bau}^{2}$

Guido Aranha Rosito ${ }^{3}$

'Departamento de Clínica Médica e Disciplina

de Nefrologia do

Curso de Medicina da

Universidade Luterana

do Brasil - ULBRA.

${ }^{2}$ ULBRA.

${ }^{3}$ Departamento de Clínica

Médica do Curso de

Medicina da ULBRA;

Universidade Federal de

Ciências da Saúde de

Porto Alegre - UFCSPA.

Data de submissão: 01/08/2011 Data de aprovação: 16/11/2011

Correspondência para:

Prof. Jayme Eduardo

Burmeister

Avenida Coronel Lucas de

Oliveira 1270, apto. 301,

Bela Vista

Porto Alegre - RS - Brasil

CEP 90440-010

E-mail: jb.nefro@gmail.com

$O$ referido estudo foi realizado na ULBRA -

Canoas - RS - Brasil.

Os autores declaram a inexistência de conflitos de interesse.

\section{Resumo}

Introdução: A causa mais frequente de insuficiência renal crônica (IRC) nos pacientes iniciando tratamento dialítico nos países desenvolvidos é o diabetes mellitus (DM), com índices crescentes e que hoje se aproximam de $50 \%$. No Brasil, os dados disponíveis indicam que essa prevalência é inferior, em torno de $27 \%$, embora venha aumentando. Objetivos: Estimar a prevalência de DM na população adulta de pacientes em tratamento hemodialítico por IRC em Porto Alegre, Brasil. Métodos: Estudo transversal descritivo, quantitativo e analítico, com inquérito sobre a prevalência de DM entre os 1.288 pacientes em tratamento por hemodiálise (HD) crônica nas 15 clínicas do município nos meses de junho e julho de 2009 . Resultados: 488 pacientes diabéticos foram identificados, uma prevalência de $37,9 \%$, variando de $21-75 \%$ nas diferentes clínicas da cidade. Conclusões: A prevalência de DM entre pacientes em HD crônica em Porto Alegre é muito superior ao que tem sido atribuído como causa de IRC no país, o que indica a possibilidade de que essa etiologia de IRC possa estar sendo subestimada. Palavras-chave: Prevalência. Diabetes mellitus. Diálise renal. Falência renal crônica.

\section{INTRODUÇÃO}

Diabetes mellitus (DM) é a principal causa de insuficiência renal crônica (IRC) em pacientes ingressando em hemodiálise (HD) nos países desenvolvidos, representando até quase $50 \%$ dos novos casos. ${ }^{1,2}$ Os registros europeus e norte-americanos têm identificado que esses números têm crescido progressivamente. ${ }^{2,3}$ Esse fenômeno, que parece ser devido, principalmente, ao

\section{Abstract}

Introduction: The most common cause of chronic renal failure (CRF) among patients who are starting on dialysis in developed countries is diabetes mellitus (DM), with growing rates approaching $50 \%$. In Brazil, the available data indicate a lower prevalence, around $27 \%$, even though it is also increasing. Objectives: To estimate the prevalence of DM in the adult population of patients on hemodialysis for CRF in Porto Alegre, Brazil. Methods: Cross-sectional, descriptive, quantitative and analytical survey study on the prevalence of DM among the 1,288 patients on hemodialysis (HD) in all the 15 clinics in the city from June to July, 2009. Results: 488 diabetic patients were identified, a prevalence of $37.9 \%$, ranging from $21-75 \%$ in the different clinics of the city. Conclusions: The prevalence of DM among chronic HD patients in Porto Alegre is higher than what was previously published as a cause of CRF in the country, which indicates the possibility that this etiology of CRF may have been underestimated.

Keywords: Prevalence. Diabetes mellitus. Renal dialysis. Kidney failure, chronic. aumento da prevalência de DM na população geral, ${ }^{4}$ vem causando preocupação na comunidade médica e, em especial, entre os nefrologistas pelas possíveis consequências futuras.

No Brasil, há algumas publicações que analisaram a prevalência de DM como causa de IRC em pacientes em HD, sendo a maioria há vários anos e com dados geralmente restritos a serviços individuais 
ou regionais. ${ }^{5-11}$ Exceções têm sido os registros da Sociedade Brasileira de Nefrologia (SBN); em 1999, um inquérito epidemiológico encontrou uma prevalência de $17 \%$ no país (com $19 \%$ na Região Sul). ${ }^{12}$ Mais recentemente, uma publicação com análise do perfil epidemiológico dos pacientes renais crônicos que iniciaram terapia renal substitutiva (TRS) no Brasil, entre 2000 e 2004, encontrou o DM como a causa da doença renal em $18 \%$ dos indivíduos. ${ }^{13}$ Já os censos brasileiros de HD promovidos pela SBN, em 2008 e 2009, estimaram essa prevalência em 25,7 e $27 \%$, respectivamente, ${ }^{14,15}$ todos esses dados sugerindo um aumento progressivo também no Brasil.

Avaliações dessa crescente prevalência de DM nos indivíduos em HD são relevantes para o planejamento em saúde pública, pois esse grupo de pacientes necessita de cuidados mais complexos, gerando maior custo, além de apresentar índices mais elevados de morbidade e mortalidade do que os não-diabéticos. ${ }^{16-18}$

O presente estudo teve por objetivo estimar a prevalência de DM nos pacientes com IRC em programa de HD na cidade de Porto Alegre, Brasil e sua distribuição pelos diferentes centros de tratamento da cidade.

\section{Métodos}

Este estudo transversal foi realizado no período de junho a julho de 2009, em todas as quinze clínicas de diálise da cidade de Porto Alegre, incluindo dados individualizados por serviço e da totalidade dos pacientes em tratamento de IRC com HD ambulatorial intermitente. $\mathrm{O}$ estudo foi aprovado pelo Comitê de Ética em Pesquisa da Universidade Luterana do Brasil - ULBRA.

Os dados foram obtidos diretamente dos profissionais médicos e de enfermagem responsáveis por cada uma das unidades de diálise da cidade. Entre 20 de junho e 15 de julho de 2009, foi estabelecido contato telefônico pelo primeiro autor deste texto com um dos responsáveis técnicos (médico ou enfermeira) de cada clínica de diálise de Porto Alegre, explicando-se o objetivo inicial do estudo - estimativa da prevalência de DM entre os pacientes em programa de HD por IRC. Foi também explicado que esse dado seria utilizado para um cálculo amostral, visando outro estudo com dados individuais sobre a prevalência de fatores de risco cardiovascular nos diabéticos em HD (Estudo CORDIAL, em andamento). Solicitou-se que fosse informado o número total de indivíduos com dezoito anos de idade ou mais que estivessem em TRS com $\mathrm{HD}$, assim como o número de pacientes com diagnóstico de DM dentro desse grupo, independente da causa definida para a IRC.

A análise estatística foi realizada pelo teste do Qui-quadrado com correção de Yates, por meio do software estatístico GraphPad inStat (GraphPad Software Inc., San Diego - CA, USA). O nível de significância estatística foi estabelecido como $\mathrm{p}<0,05$.

\section{Resultados}

No período do estudo - entre junho e julho de 2009 - 1.288 pacientes renais crônicos foram informados como estando em tratamento com HD nas quinze diferentes clínicas da cidade de Porto Alegre. Foram identificados 488 diabéticos - uma prevalência de $37,9 \%$, variando de 21 a $75 \%$ nos diferentes centros.

A Tabela 1 apresenta a distribuição dos pacientes agrupados de acordo com os locais de tratamento (centros hospitalares de diálise ou clínicas autônomas) e de acordo com a instituição responsável pelo financiamento do tratamento - Sistema Único de Saúde (SUS) ou planos de saúde. A Tabela 2 mostra a

\begin{tabular}{|c|c|c|c|c|}
\hline Tabela 1 & \multicolumn{4}{|c|}{$\begin{array}{l}\text { DISTRIBUIÇÃO DOS PACIENTES E PREVALÊNCIA DE DIABETES MELLITUS NOS DIFERENTES TIPOS DE SERVIÇO DE } \\
\text { HEMODIÁLISE DA CIDADE }\end{array}$} \\
\hline \multicolumn{2}{|c|}{ Tipos de Serviço } & $\begin{array}{c}\text { Total } \\
\mathrm{n}\end{array}$ & $\underset{n}{\mathrm{DM}}$ & $\begin{array}{c}\text { Prevalência DM } \\
\%\end{array}$ \\
\hline \multicolumn{2}{|c|}{ Hospitais universitários - SUS } & 331 & 88 & 26,6 \\
\hline \multicolumn{2}{|c|}{ Hospitais públicos - SUS } & 75 & 21 & 28,0 \\
\hline \multicolumn{2}{|c|}{ Hospitais privados - SUS } & 289 & 103 & 35,6 \\
\hline \multicolumn{2}{|c|}{ Hospitais privados - planos de saúde } & 111 & 63 & 56,8 \\
\hline \multicolumn{2}{|c|}{ Clínicas autônomas - SUS } & 457 & 208 & 45,5 \\
\hline \multicolumn{2}{|c|}{ Clínicas autônomas - planos de saúde } & 25 & 5 & 20,0 \\
\hline \multicolumn{2}{|l|}{ Total geral } & 1.288 & 488 & 37,9 \\
\hline
\end{tabular}

SUS: Sistema Único de Saúde; DM: diabetes mellitus. 
distribuição da população do estudo nos quinze centros de diálise existentes na cidade neste período.

O Gráfico 1 mostra um comparativo dos centros que atendem exclusivamente pacientes de planos de saúde (68 diabéticos dentre 136 indivíduos - prevalência de $50,0 \%$ ) com os demais centros cujos pacientes são majoritariamente do SUS (420 diabéticos dentre 1.152 pacientes - prevalência de $36,5 \%)$, observando-se uma diferença significativa $(\mathrm{p}=0,0028)$.

O Gráfico 2 compara os centros de diálise em hospitais (275 diabéticos entre 806 pacientes - prevalência de $34,1 \%$ ) com as clínicas autônomas (213 diabéticos entre 482 indivíduos - prevalência de 44,2\%) $-p=0,0004$. Essa diferença se torna ainda mais notável se comparados apenas aqueles que fazem atendimento pelo SUS: 212 diabéticos entre 695 pacientes em hospitais (prevalência de 30,5\%) versus 208 diabéticos entre 457 pacientes nas clínicas (prevalência de 45,5\%) - p = 0,0001 (Gráfico 3).

O Gráfico 4 compara os centros universitários hospitalares que atendem principalmente pacientes do SUS (88 diabéticos entre 331 pacientes - prevalência de $26,6 \%$ ) com os demais serviços com pacientes do SUS (332 diabéticos em 821 indivíduos - prevalência de $40,4 \%)-p=0,0001$.

\begin{tabular}{|c|c|c|c|}
\hline \multirow{2}{*}{$\begin{array}{l}\text { Tabela } 2 \\
\text { Serviços de } \\
\text { Hemodiálise }\end{array}$} & \multicolumn{3}{|c|}{$\begin{array}{l}\text { TOTAL DE PACIENTES EM HEMODIÁLISE E } \\
\text { PREVALÊNCIA DE DIABETES MELLITUS NOS } \\
\text { QUINZE CENTROS DE DIÁLISE DA CIDADE }\end{array}$} \\
\hline & $\begin{array}{c}\text { Total } \\
\mathrm{n}\end{array}$ & $\begin{array}{c}\mathrm{DM} \\
\mathrm{n}\end{array}$ & $\begin{array}{c}\text { Prevalência DM } \\
\%\end{array}$ \\
\hline$A$ & 57 & 17 & 29,8 \\
\hline B & 73 & 28 & 38,4 \\
\hline C & 201 & 43 & 21,4 \\
\hline D & 75 & 21 & 28,0 \\
\hline E & 43 & 13 & 30,2 \\
\hline $\mathrm{F}$ & 89 & 38 & 42,7 \\
\hline G & 64 & 15 & 23,4 \\
\hline $\mathrm{H}$ & 93 & 37 & 39,8 \\
\hline । & 41 & 31 & 75,6 \\
\hline$J$ & 70 & 32 & 45,7 \\
\hline K & 112 & 46 & 41,1 \\
\hline L & 186 & 103 & 55,4 \\
\hline M & 43 & 21 & 48,8 \\
\hline$N$ & 116 & 38 & 32,8 \\
\hline $\mathrm{O}$ & 25 & 5 & 20,0 \\
\hline Total geral & 1.288 & 488 & 37,9 \\
\hline
\end{tabular}

DM: diabetes mellitus.
Gráfico 1. Prevalência de diabetes mellitus nos serviços de hemodiálise com pacientes de planos de saúde e do Sistema Único de Saúde $(p=0,028)$.

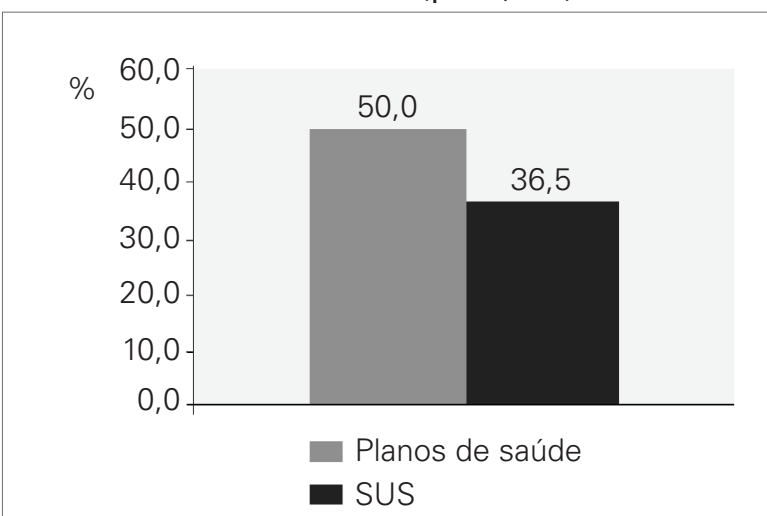

SUS: Sistema Único de Saúde.

Gráfico 2. Prevalência de diabetes mellitus nos serviços intra-hospitalares e nas clínicas autônomas $(p=0,0004)$.

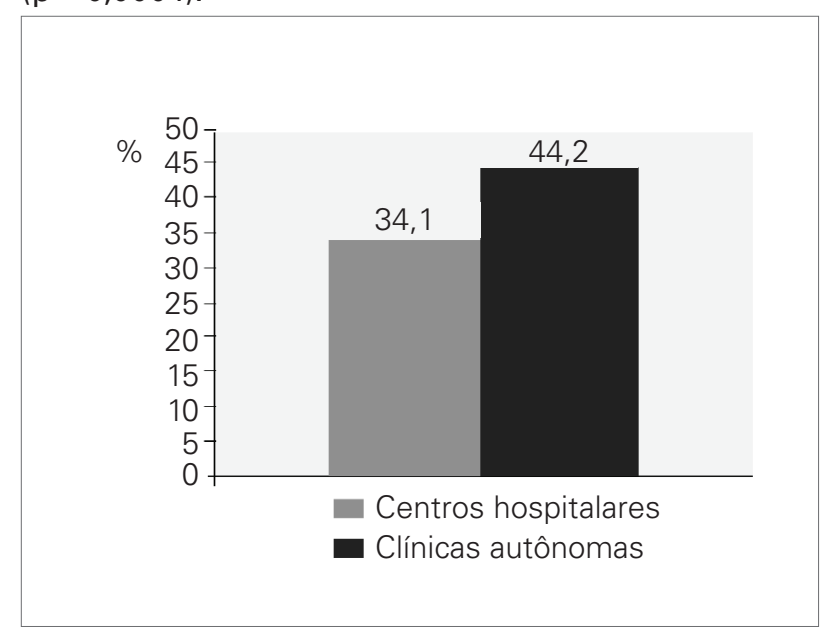

Gráfico 3. Prevalência de diabetes mellitus nos pacientes do Sistema Único de Saúde, serviços intrahospitalares e clínicas autônomas $(p=0,0001)$.

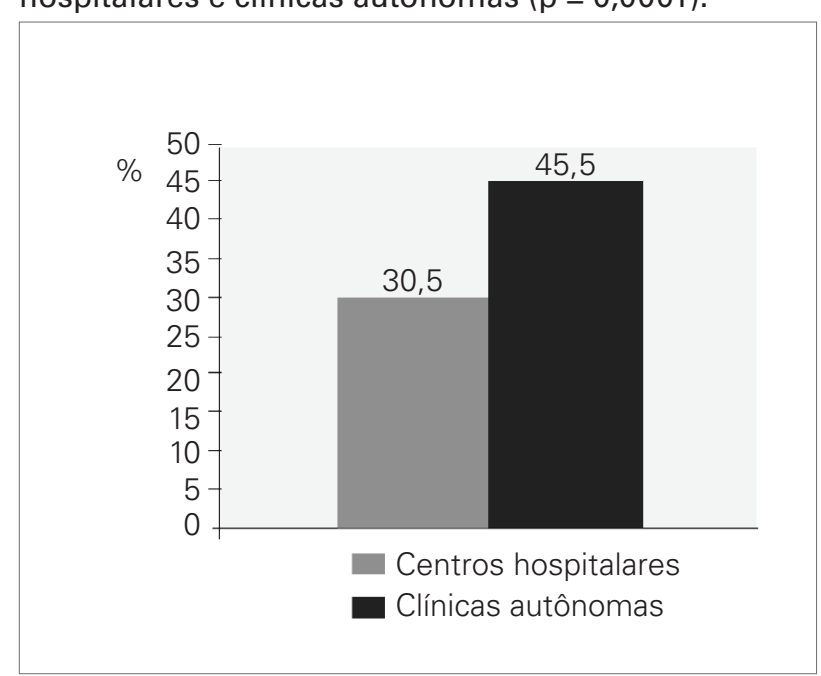


Gráfico 4. Prevalência de diabetes mellitus nos pacientes do Sistema Único de Saúde, hospitais universitários e demais hospitais/clínicas $(p=0,0001)$.

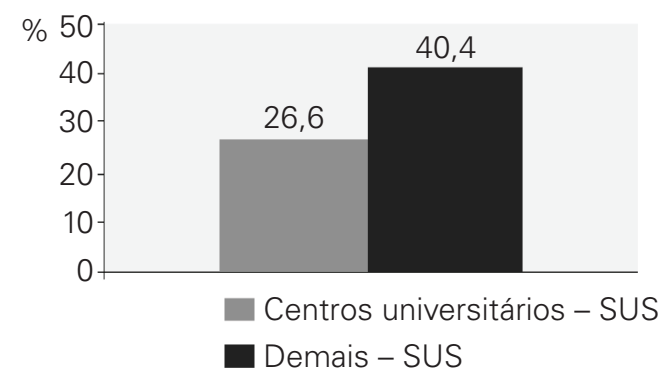

SUS: Sistema Único de Saúde.

\section{Discussão}

O achado de quase $40 \%$ de diabéticos entre os renais crônicos em HD na cidade é de grande relevância. Considerando os dados anteriores disponíveis para o país, parece haver um aumento impactante, sendo comparável ao encontrado em populações urbanas dos países desenvolvidos. Esse índice mais elevado de prevalência em relação a estudos prévios se deve a diversos fatores - entre alguns, a possibilidade de um fenômeno regional, já que obesidade e DM apresentam maior prevalência na Região Sul do Brasili: ${ }^{19,20}$ uma redução da mortalidade dos pacientes diabéticos em HD, fenômeno já observado em outros países; ${ }^{3}$ a presença de pacientes mais idosos com DM2 (um reflexo, pelo menos parcialmente, da maior expectativa de vida dos diabéticos na população geral); ${ }^{21}$ e o aumento da obesidade na população geral. ${ }^{22}$

Todos os registros anteriores disponíveis sobre a prevalência de DM em HD no Brasil apresentam prevalências de $\mathrm{DM}$ menores do que a nossa. Além dos estudos locais ou regionais, cabe ressaltar aqueles que abrangeram todo o país. Cherchiglia et al. ${ }^{13}$ encontraram prevalência de $15 \%$ de DM como causa da doença renal entre todos os pacientes que iniciaram TRS com HD no Brasil nos anos de 2000 a 2004. Esse estudo baseou-se nos registros administrativos do banco de dados das Autorizações de Procedimentos de Alta Complexidade (APAC) do Sistema de Informações Ambulatoriais (SIA) do SUS, onde são inseridos no momento da entrada de cada paciente no tratamento dialítico do SUS. No entanto, esse índice pode estar subestimado, pois os registros desse sistema apontam para "causa indeterminada" como motivo da doença renal crônica (DRC) em 43\% dos casos iniciando tratamento com HD, um número bastante elevado e que, provavelmente, traduz a não identificação inicial da causa da DRC podendo, portanto, não representar a realidade clínica. Além disso, o registro do SIA/SUS não inclui os pacientes em HD crônica subsidiados por planos de saúde, um grupo que corresponde a 13,3\% do total no país, segundo estimativa da SBN. ${ }^{15}$

Já o Censo Brasileiro de Diálise da SBN, em 2009, encontrou uma prevalência de $27 \%$ para DM em HD no país. ${ }^{15}$ Considerando-se que o resultado do presente estudo, de 37,9\% em Porto Alegre, foi desse mesmo ano de 2009, é notável e relevante a diferença, sendo bastante provável a existência de fatores locais e/ou regionais para justificá-la. Por um lado, como o Censo da SBN não apresenta dados por região, qualquer análise comparativa fica impossibilitada; por outro, a prevalência significativamente menor de DM nos centros de diálise de Porto Alegre que atendem pacientes do SUS (comparados com os que atendem apenas pacientes de planos de saúde) (Gráfico 1), assim como o mesmo achado, quando se comparam centros hospitalares com as clínicas autônomas (Gráficos 2 e 3) e, também, os centros universitários com os demais centros de diálise SUS (Gráfico 4), necessita de análise mais específica em busca de justificativas.

$\mathrm{O}$ estudo apresentou algumas limitações, a saber: a forma de obtenção dos dados foi por informação telefônica por meio dos profissionais responsáveis em cada serviço, ao invés de identificação individual da situação de cada paciente in loco; também não se buscou identificar casos em que a causa da DRC possa ter sido outra que não a nefropatia diabética, embora o dado epidemiológico mais relevante seja a própria presença do DM.

Em conclusão, a prevalência de diabetes mellitus entre a população de renais crônicos adultos em HD na cidade de Porto Alegre aproxima-se de 40\%, sendo maior do que a verificada em outros estudos anteriores no Brasil. Essa prevalência é bastante superior àquela que tem sido atribuída como causa da IRC entre os pacientes em HD no país. Há necessidade de novos e cuidadosos estudos epidemiológicos que abordem a crescente contribuição da DM na IRC.

\section{REFERÊNCIAS}

1. Locatelli F, Pozzoni P, Vecchio LD. Renal replacement therapy in patients with diabetes and end-stage renal disease. J Am Soc Nephrol 2004;15 Suppl:S25-9.

2. United States Renal Data System (USRDS). Excerpts from 2009. Annual data report: Atlas of end-stage renal disease in the United States. Am J Kidney Dis 2010;55 Suppl:S1.

3. Van Dijk PC, Jager KJ, Stengel B, et al. Renal replacement therapy for diabetic end-stage renal 
disease: data from 10 registries in Europe (1991-2000). Kidney Int 2005;67:1489-99.

4. Bommer J. Prevalence and socio-economic aspects of chronic kidney disease. Nephrol Dial Transplant 2002;17 Suppl:8-12.

5. Antero DC, Antero MAJ, Junkes SA, et al. Nefropatia diabética nas unidades de diálise da Região Sul de Santa Catarina: perfil clínico-epidemiologico. ACM Arq Catarin Med 2008;37:70-5.

6. Peres LAB, Matsuo $\mathrm{T}$, Delfino $\mathrm{V}$, et al. Aumento na prevalência de diabete melito como causa de insuficiência renal crônica dialítica: análise de 20 anos na Região Oeste do Paraná. Arq Bras Endocrinol Metabol 2007;51:111-5.

7. Bohlke M, Colla TG, Silveira CA, et al. Análise de sobrevida do diabético em centro brasileiro de diálise. J Bras Nefrol 2002;24:7-11.

8. Bruno RM. Pacientes diabéticos em diálise: características clínicas, sobrevida e fatores prognósticos. [dissertation]. Rio Grande do Sul: Universidade Federal do Rio Grande do Sul; 1999.

9. Pinto FM, Anção MS, Sakumoto M, et al. Contribuição da nefropatia diabética para a insuficiência renal crônica na Grande São Paulo. J Bras Nefrol 1997;19:256-63.

10. Sesso R, Melaragno CS, Luconi PS, et al. Sobrevida de pacientes diabéticos em diálise. Rev Assoc Med Bras 1995; 41:178-82.

11. Sesso R, Anção M, Madeira AS. Aspectos epidemiológicos do tratamento dialítico na Grande São Paulo. Rev Assoc Med Bras 1994;40:10-4.

12. Sesso R. Inquérito epidemiológico em unidades de diálise do Brasil. J Bras Nefrol 2000;22 Suppl:23-6.

13. Cherchiglia ML, Machado EL, Szuster DAC, et al. Epidemiological profile of patients on renal replacement therapy in Brazil, 2000-2004. Rev Saúde Pública 2010;44:1-10

14. Sesso R, Lopes AA, Thomé FS, et al. Relatório do Censo Brasileiro de Diálise, 2008. J Bras Nefrol 2008;30:233-8

15. Sesso R, Lopes AA, Thomé FS, et al. Censo Brasileiro de Diálise, 2009. J Bras Nefrol 2010;32:380-4.

16. United States Renal Data System (USRDS). Excerpts from 2008. Annual data report: Atlas of end-stage renal disease in the United States. Am J Kidney Dis 2009; Suppl:S1-S5.

17. Wolfe RA, Gaylin DS, Port FK, et al. Using USRDS generated mortality tables to compare local ESRD mortality rates to national rates. Kidney Int 1992;42:991-6.

18. United States Renal Data System (USRDS). Excerpts from 2005. Annual data report: Atlas of end-stage renal disease in the United States. Am J Kidney Dis 2006;47 Suppl:S1-S2.

19. Ferreira VA, Magalhães R. Obesidade no Brasil: tendências atuais. Rev Port Saúde Pública 2006;24:71-81.

20. Malerbi DA, Franco LJ. Multicenter study of the prevalence of diabetes mellitus and impaired glucose tolerance in the urban Brazilian population aged 30-69 year. The Brazilian Cooperative Group on the Study of Diabetes Prevalence. Diabetes Care 1992;15:1509-16.

21. Picolli GB, Quarello F, Bonello F, et al. Diabetic patients on dialysis: a changing picture. Kidney Int Suppl 1993;41:S14-7.

22. Wild S, Roglic G, Green A, et al. Global prevalence of diabetes: estimates for the year 2000 and projections for 2030. Diabetes Care 2004;27:1047-53. 